\title{
Multiagent Decision Making and Learning in Urban Environments
}

\author{
Akshat Kumar \\ School of Information Systems, Singapore Management University \\ akshatkumar@smu.edu.sg
}

\begin{abstract}
Our increasingly interconnected urban environments provide several opportunities to deploy intelligent agents-from self-driving cars, ships to aerial drones - that promise to radically improve productivity and safety. Achieving coordination among agents in such urban settings presents several algorithmic challenges-ability to scale to thousands of agents, addressing uncertainty, and partial observability in the environment. In addition, accurate domain models need to be learned from data that is often noisy and available only at an aggregate level. In this paper, I will overview some of our recent contributions towards developing planning and reinforcement learning strategies to address several such challenges present in largescale urban multiagent systems.
\end{abstract}

\section{Introduction}

Our society and urban environments are rapidly getting interconnected by the internet of things (IoT). A number of smart devices embedded in everyday objects are capable of sensing their environment, and taking decisions to increase our productivity, safety and efficiency. As an example, autonomous self-driving cars are able to perceive their environment, and interact with each other to create future applications such as smart traffic light intersections [Au et al., 2016]. Similarly, for maritime traffic, e-navigation aims to improve the management of the sea traffic by digitizing both on-board marine information and the communication between vessels and maritime traffic control authorities ${ }^{1}$. Such e-navigation would pave the way for autonomous vessels, and has tremendous potential to improve coordination among vessels to reduce congestion and improve safety of navigation in busy ports of the world [Agussurja et al., 2018]. There is no lack of such interconnected urban environments where learning and modeling interactions among agents (which may represent self-driving cars and trucks, autonomous vessels, drones) is the key to enable the overall productivity and safety of the resulting large multiagent system.

\footnotetext{
${ }^{1}$ http://www.imo.org/en/OurWork/Safety/Navigation/Pages/ eNavigation.aspx
}

Our recent work is directed towards modeling such large urban systems, and developing scalable planning and reinforcement learning (RL) based approaches that enable effective coordination among agents. Decentralized partially observable MDP (Dec-POMDPs) have emerged as a popular framework for modeling such multiagent sequential decision making problems under uncertainty [Bernstein et al., 2002; Kumar and Zilberstein, 2009; Amato et al., 2010; Kumar et al., 2015; Kumar et al., 2016]. However, it is known to be challenging (NEXP-Hard complexity) even for the smallest two-agent systems [Bernstein et al., 2002]. To address the complexity, various models are explored where agent interactions are limited by design by enforcing various conditional and contextual independencies such as transition and observation independence among agents [Nair et al., 2005] where agents are coupled primarily via joint-rewards, event driven interactions [Becker et al., 2004], and weakly coupled agents [Spaan and Melo, 2008].

Our contributions. Previous such models and algorithms in multiagent decision making have been either relatively general but not very scalable, or relatively scalable but with limited applicability. Our recent and ongoing work challenges this current state of affairs by proposing new models and algorithms that (a) are applicable to a wide range of problems of practical importance, particularly in urban system optimization, (b) lead to scalable algorithms for coordinating thousands of agents in settings where agents partially observe their environment, and there is uncertainty present, and (c) constructing faithful domain simulators for different urban settings (e.g., taxi fleet optimization, maritime traffic management) learned from real world historical data. A key part of our innovation involves using graphical models and probabilistic inference to learn models of urban systems, and the development of new reinforcement learning strategies, that allow agents to rapidly discover more efficient decisions through feedback on past behavioral outcomes using a domain simulator.

\section{The Dec-POMDP Model}

A Dec-POMDP generalizes single agent Markov decision process to account for multiple agents operating in the environment. A distinguishing feature is that agents observe their environment and other agents only partially. Based on 
the local information agents receive (which may be different for different agents), each agent chooses the next action to take (in parallel) operating in a sequential manner over a finite or an infinite horizon. At each time step, the agent-team also obtains a joint-reward. The goal is to compute policies (mapping from local observation history to actions for each agent) to maximize the total reward over the planning horizon. The joint-reward makes the problem cooperative, and action selection based on local observations makes the problem decentralized.

A Dec-POMDP can be defined by a tuple $\left\langle I, S,\left\{A^{i}\right\}, P, R,\left\{Y^{i}\right\}, O, \gamma\right\rangle$, where $I$ denotes a finite set of $n$ agents; $S$ denotes a finite set of states with designated initial state distribution $\eta_{0} ; A^{i}$ denotes a finite set of actions for each agent $i ; P$ denotes state transition probabilities: $P\left(s^{\prime} \mid s, \vec{a}\right)$, the probability of transitioning from state $s$ to $s^{\prime}$ when the joint-action $\vec{a}$ is taken by the agents; $R$ denotes the reward function: $R(s, \vec{a})$ is the immediate reward for being in state $s$ and joint-action taken as $\vec{a} ; Y^{i}$ denotes a finite set of observations for each agent $i$; $O$ denotes the observation probabilities: $O\left(\vec{y} \mid s^{\prime}, \vec{a}\right)$ is the probability of receiving the joint-observation $\vec{y}$ when the last joint-action taken was $\vec{a}$ that resulted in the environment state being $s^{\prime} ; \gamma$ denotes the reward discounting factor. An agent $i$ 's policy, $\boldsymbol{\theta}^{i}: \bar{Y}^{i} \rightarrow A^{i}$, maps the set of all possible observation histories $\bar{Y}^{i}$ to actions. Solving a Dec-POMDP entails finding the joint-policy $\boldsymbol{\theta}=\left\langle\boldsymbol{\theta}^{1}, \ldots, \boldsymbol{\theta}^{n}\right\rangle$ that maximizes the total expected reward:

$$
\mathbb{E}\left[\sum_{t=0}^{\infty} \gamma^{t} R\left(s_{t}, \vec{a}_{t} ; \boldsymbol{\theta}\right)\right]
$$

where $\boldsymbol{\theta}$ denotes the joint-policy and subscript $t$ denotes the dependence on time. There are several representations possible for local policies $\theta^{i}$ such as policy trees, finite-state controllers [Amato et al., 2010; Kumar et al., 2015], and deep neural networks [Nguyen et al., 2018].

\section{Modeling Urban Environments}

Several urban environments can be modeled as a diffusion, cascade or flow of entities (e.g., vehicles, vessels, humans) over an underlying geographical network [Kumar et al., 2013]. For example, traffic flow can be modeled as diffusion of vehicles over the network [Kumar et al., 2013], maritime traffic as vessel movement between sea zones [Singh et al., 2019], and people flow over a geographical area [Iwata and Shimizu, 2019]. However, in many such domains, individual data tracking the movement of each entity is either not available (e.g., to protect privacy of individuals) or too expensive to collect. Only the aggregate or collective data (which may be noisy or missing) is observed. For example, consider a road traffic network. A key learning problem in such traffic networks is estimating the turn probabilites for each road segment of this network [Kumar et al., 2013]. Several popular analytical models of traffic flow such as the cell transmission model [Daganzo, 1994] are based on the assumption that turn probabilities are known a priori for each location. In several urban traffic networks, aggregate data in the form of vehicle count is already collected for each road segment using

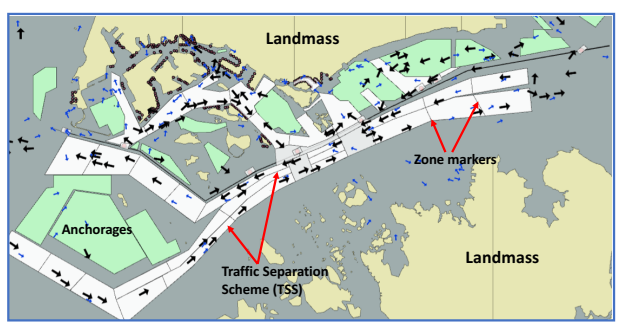

(a)

Figure 1: Electronic navigation chart (ENC) of straits near a large asian city with color-coded features

inductive-loop traffic detectors, and we show that such aggregate level information is sufficient to learn turn probabilities for traffic networks and model the traffic flow [Kumar et al., 2013].

As another example, figure 1 shows the e-navigation chart (ENC) of a strait [Singh et al., 2019]. The ENC is composed of several features such as anchorages where vessels anchor and wait for services, berths, pilot boarding grounds, and the traffic separation scheme or TSS. The TSS (figure 1) is the set of mandatory unidirectional routes designed to carry bulk of the maritime traffic to reduce collision risk among vessels transitioning through or entering the Straits. Based on geographical features, the TSS can be further divided into smaller zones, and maritime traffic can be thought of as flow of vessels over zones. In the maritime case, although individual vessel trajectories are available, modeling the precise movement of each vessel is intractable (it requires modeling interaction with other vessels, effects of weather on the movement among other factors). Therefore, modeling the traffic at the aggregate level of zones (where we observe how many vessels are present in which zone at each time step) is significantly more tractable, and in our empirical tests, we show such an aggregate modeling is accurate enough to replicate historical patterns [Singh et al., 2019].

\subsection{Collective Graphical Models and Learning Domain Simulators}

We use the framework of collective graphical models (CGMs) to model several types of urban environments [Sheldon and Dietterich, 2011] where we fit a model of the behavior of individuals but our data consist only of aggregate information or counts. CGMs compactly describe the distribution of the aggregate statistics of a population sampled independently from a discrete graphical model. Let $G=(V, E)$ denote an undirected graph. Consider the following pairwise graphical model over the discrete random vector $\boldsymbol{X}=\left(X_{1}, \ldots, X_{|V|}\right)$ :

$$
p(\mathbf{x} ; \boldsymbol{\theta})=\operatorname{Pr}(\boldsymbol{X}=\mathbf{x} ; \boldsymbol{\theta})=\frac{1}{Z(\boldsymbol{\theta})} \prod_{(i, j) \in E} \phi_{i j}\left(x_{i}, x_{j} ; \boldsymbol{\theta}\right) .
$$

Here, $\phi_{i j}(\cdot, \cdot ; \boldsymbol{\theta})$ is a local potential defined on the setting of variables $\left(X_{i}, X_{j}\right)$. The local potentials depend on the parameter vector $\boldsymbol{\theta}$, and $Z(\boldsymbol{\theta})$ is the partition function. We assume that each variable $X_{i}$ takes values in the same finite set $\mathcal{X}$. Now, consider an ordered sample $\mathbf{x}^{(1)}, \ldots, \mathbf{x}^{(M)}$ of random vectors drawn independently from the graphical model. 


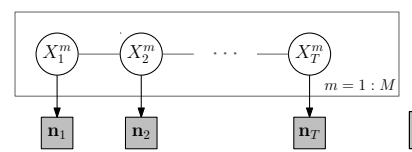

(a)

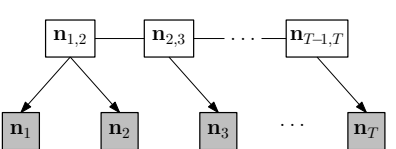

(b)
Figure 2: CGM representation using plate notation. (a) shows dependence of count tables $\mathbf{n}$ on individuals; (b) shows resulting CGM after marginalizing out all individuals

We also refer to this sample as a population (of size $M$ ). We define the contingency tables $\mathbf{n}_{i}=\left(n_{i}\left(x_{i}\right): x_{i} \in \mathcal{X}\right)$ over nodes of the model and $\mathbf{n}_{i j}=\left(n_{i j}\left(x_{i}, x_{j}\right): x_{i}, x_{j} \in \mathcal{X}\right)$ over edges of the model, whose entries count the number of times particular variable settings occur in the population. Define the vector $\mathbf{n}$ to be the concatenation of all edge-based contingency tables $\mathbf{n}_{i j}$ together with all node-based contingency tables $\mathbf{n}_{i}$. This is a random vector that depends on the entire population and comprises sufficient statistics of the population, which can be seen by writing the joint probability:

$$
\begin{aligned}
& p\left(\mathbf{x}^{(1)}, \ldots, \mathbf{x}^{(M)} ; \boldsymbol{\theta}\right)=g(\mathbf{n}, \boldsymbol{\theta})= \\
& \quad=\frac{1}{Z(\boldsymbol{\theta})^{M}} \prod_{(i, j) \in E} \prod_{x_{i}, x_{j}} \phi_{i j}\left(x_{i}, x_{j} ; \boldsymbol{\theta}\right)^{n_{i j}\left(x_{i}, x_{j}\right)} .
\end{aligned}
$$

In CGMs, one makes noisy observations $\mathbf{y}$ of some subset of the sufficient statistics $\mathbf{n}$ and then seeks to answer queries about the sufficient statistics given y (e.g., for the purpose of learning the parameters $\boldsymbol{\theta}$ ) through the conditional distribution $p(\mathbf{n} \mid \mathbf{y} ; \boldsymbol{\theta}) \propto p(\mathbf{n} ; \boldsymbol{\theta}) p(\mathbf{y} \mid \mathbf{n})$. The first term in this product, $p(\mathbf{n} ; \boldsymbol{\theta})$, is the prior distribution over the sufficient statistics or the CGM distribution. Its exact form is shown in [Sheldon et al., 2013]. The second term, $p(\mathbf{y} \mid \mathbf{n})$, is the noise model. Figure 2 shows a graphical representation of a CGM over a chain structured individual model. We have worked on developing several probabilisitic inference based approaches to develop message-passing algorithms to compute the maximum-a-posteriori assignment (or the most likely $\mathbf{n}$ given the noisy observations $\mathbf{y}$ ) and learning the parameters $\boldsymbol{\theta}$ using maximum likelihood estimation [Sheldon et al., 2013; Kumar et al., 2013; Sun et al., 2015; Nguyen et al., 2016]. CGMs are an ideal formalism to represent urban domains such as traffic. We have used models based on CGMs to represent the maritime traffic and learned parameters of such models using historical aggregate data [Singh et al., 2019]. We envision that constructing domain simulators based on such aggregate modeling of data would be crucial for scaling up learning and decision making in large scale urban environments as CGMs provide a tractable representation to model a large number of agents.

\subsection{Collective Multiagent Decision Making}

Our recent work focuses towards developing general decision theoretic frameworks for collective multiagent decision making that allow to control the behavior of a population of nearly identical agents operating collaboratively in an uncertain and partially observable environment. Our key enabling insight and related assumption is that in several urban environments

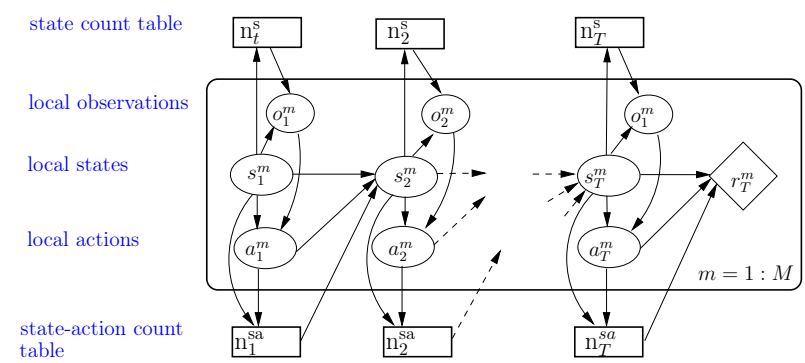

(a) Dynamic Bayes net for Collective Dec-POMDP model

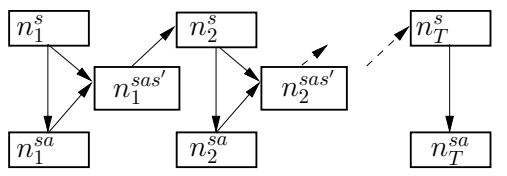

(b) Collective Dec-POMDP model $a f$ ter marginalizing out individual agents

Figure 3: The $\mathbb{C D e c}-$ POMDP model

(such as transportation, supply-demand matching) agent interactions are governed by the aggregate count and types of agents, and do not depend on the specific identities of individual agents. This insight makes it possible to construct scalable and general approaches to multiagent modeling, simulation and optimization that are capable of addressing a range of practical problems in urban systems. Such modeling also addresses shortcomings of previous multiagent planning approaches which are either general but not scalable or scalable but with very limited applicability.

To formalize such collective decision making problems, we have recently developed the framework of $\mathbb{C D e c}$ POMDP [Nguyen et al., 2017a; Nguyen et al., 2017b; Nguyen et al., 2018] or collective decentralized POMDPs. The $\mathbb{C D e c}-P O M D P$ model is based on the idea of partial exchangeability [Diaconis and Freedman, 1980; Niepert and Van den Broeck, 2014], and collective graphical models [Sheldon and Dietterich, 2011; Sun et al., 2015]. Partial exchangeability in probabilistic inference is complementary to the notion of conditional and contextual independence, and combining all of them leads to a larger class of tractable models and inference algorithms [Niepert and Van den Broeck, 2014]. Previous works in multiagent planning have mostly explored only conditional and contextual independences in multiagent models [Nair et al., 2005; Witwicki and Durfee, 2010]. CDec-POMDPs combine both conditional independences and partial exchangeability to solve much larger instances of multiagent decision making.

Figure 3(a) shows how different agents $m$ in a population of $M$ agents interact with each other. We assume that different agents share the state space $S$. E.g., in a transportation network, agents move in different zones of the same city. An agent $m$ 's local state at time $t$ is denoted by $s_{t}^{m}$. The local states of all the agents are aggregated to form the state count table $\mathbf{n}_{t}^{s}$ which simply counts how many agents are present in each state $i \in S$. Based on its local state and the state count table, an agent $i$ receives its local observation $o_{t}^{m}$, which it 


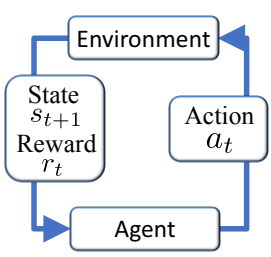

(a) Single agent RL

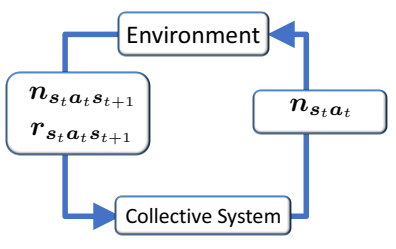

(b) Collective MARL
Figure 4: Settings for single agent and collective multiagent RL

uses to take the next action $a_{t}^{m}$ (using the policy $\pi$ ). Based on the joint states and actions of all the agents, the state-action count table $\mathbf{n}_{t}^{s a}$ is generated, which simply counts how many agents in state $i$ took action $j$ (for each $i \in S$ and $j \in A$ ). As a result of joint actions, the environment transitions to the next state, and a reward is given per agent. This particular model is equivalent to the individual model similar to figure 2(a).

Scalability. In urban settings, we may have thousands of agents. Sampling individual trajectories of each agent would be computationally intractable. Therefore, we exploit similar properties as in CGMs, and marginalize away individual agents to arrive at the collective planning model in figure 3(b) (analogous to figure 2(b)). This model only consists of count tables, and similar to CGMs, we show how to define a distribution $p(\mathbf{n})$ over these count tables [Nguyen et al., 2017a]. Notice that sampling from $p(\mathbf{n})$ is highly scalable as the dimensions of count tables do not depend on the population size $M$. Therefore, the $\mathbb{C D e c}-\mathrm{POMDP}$ model is able to effectively reason about a large population of agents.

\section{Solution Approaches}

Planning-as-inference. We have developed different types of solution approaches for computing policies for agents in the $\mathbb{C D e c}$-POMDP model. One direction is based on the planning-as-inference strategy [Toussaint and Storkey, 2006] where we cast the planning problem to that of likelihood maximization (LM) problem in a graphical model [Nguyen et $a l ., 2017 \mathrm{a}]$. We have explore extensively such planning-asinference strategy for multiagent decision making in several contexts [Kumar and Zilberstein, 2010; Kumar et al., 2011; Kumar et al., 2015; Ghosh et al., 2015; Singh and Kumar, 2019]. The main benefit of this strategy is that it opens the door to the application of machine learning approaches to planning. We have used a popular LM approach ExpectationMaximization (EM) for multiagent decision making. A key benefit of EM is that its updates often take the form of message-passing among agents, and are thus highly scalable for large multiagent systems.

Multiagent RL. Another direction we have explored for solving $\mathbb{C D e c}-\mathrm{POMDPs}$ is using multiagent RL (MARL). The MARL approaches are useful in settings when only the access to domain simulator is available, which is a fairly common setting for several urban environments. There exist several previous MARL approaches such as independent Q-learning, counterfactual multiagent policy gradients and actor-critic methods [Foerster et al., 2018; Lowe et al., 2017], and SARSA-based MARL for Dec-POMDPs [Dibangoye and Buffet, 2018]. However, most of these approaches are limited to few dozens of agents in contrast to the collective setting with thousands of agents, which is our goal.

Lifted multiagent RL. The key idea that our MARL approaches exploit is to lift the RL algorithms to work with count-based representations. We show how to define actionvalue function and value function over count tables, and prove that they are sufficient statistic for planning and RL for $\mathbb{C D e c}$ POMDPs [Nguyen et al., 2017a; Nguyen et al., 2018]. As shown in figure 4(b), the environment directly generates different count tables and the associated reward by exploiting the CGM-like distribution defined over the graphical model in figure 3(b). As a result, our RL methods do not have to sample individual agent trajectories that would have been prohibitively expensive. In addition, there are two main challenges we address for collective MARL-multiagent credit assignment (actions of which agents were more/less important), and computing low variance policy gradient estimates for faster convergence to high quality solutions even with thousands of agents. Without addressing these issues, standard policy gradient based approaches do not converge at all.

\section{Conclusion}

We have developed several approaches for achieving coordination in large multiagent systems that are increasingly becoming common in our urban environments. Our work includes representing urban domains using collective graphical models that exploit the property that agent interactions in several urban settings depend on their aggregate effects rather than their identities. We have developed several domain simulators for urban transportation settings (such as maritime traffic). We have used these simulators to develop efficient and scalable multiagent RL approaches that exploit such aggregate nature of interaction among agents. We have also addressed several challenges that arise when doing planning and RL with thousands of agents such as multiagent credit assignment and low variance gradient estimates.

\section{Acknowledgments}

I thank my collaborators and mentors. I also thank the UNiCEN center at SMU (https://unicen.smu.edu.sg/) for providing a conducive environment. The author is supported by the Singapore Ministry of Education Academic Research Fund (AcRF) Tier 2 grant MOE2018-T2-1-179.

\section{References}

[Agussurja et al., 2018] Lucas Agussurja, Akshat Kumar, and Hoong Chuin Lau. Resource-constrained scheduling for maritime traffic management. In AAAI Conference on Artificial Intelligence, pages 6086-6093, 2018.

[Amato et al., 2010] Christopher Amato, Daniel S. Bernstein, and Shlomo Zilberstein. Optimizing fixed-size stochastic controllers for pomdps and decentralized pomdps. Autonomous Agents and Multi-Agent Systems, 21(3):293-320, 2010.

[Au et al., 2016] Tsz-Chiu Au, Shun Zhang, and Peter Stone. Autonomous intersection management for semi-autonomous vehicles. In Dusan Teodorovi'c, editor, Handbook of Transportation, pages 88-104. Routledge, 2016. 
[Becker et al., 2004] Raphen Becker, Shlomo Zilberstein, and Victor Lesser. Decentralized Markov decision processes with eventdriven interactions. In International Conference on Autonomous Agents and Multiagent Systems, pages 302-309, 2004.

[Bernstein et al., 2002] Daniel S. Bernstein, Rob Givan, Neil Immerman, and Shlomo Zilberstein. The complexity of decentralized control of Markov decision processes. Mathematics of $\mathrm{Op}$ erations Research, 27:819-840, 2002.

[Daganzo, 1994] C.F. Daganzo. The cell transmission model: A dynamic representation of highway traffic consistent with the hydrodynamic theory. Transportation Research Part B: Methodological, 28(4):269-287, 1994.

[Diaconis and Freedman, 1980] P Diaconis and Diaconis Freedman. De Finetti's generalizations of exchangeability. Studies in Inductive Logic and Probability, 2:233-249, 1980.

[Dibangoye and Buffet, 2018] Jilles Steeve Dibangoye and Olivier Buffet. Learning to act in decentralized partially observable MDPs. In International Conference on Machine Learning, pages 1241-1250, 2018.

[Foerster et al., 2018] Jakob Foerster, Gregory Farquhar, Triantafyllos Afouras, Nantas Nardelli, and Shimon Whiteson. Counterfactual multi-agent policy gradients. In AAAI Conference on Artificial Intelligence, 2018.

[Ghosh et al., 2015] Supriyo Ghosh, Akshat Kumar, and Pradeep Varakantham. Probabilistic inference based message-passing for resource constrained DCOPs. In International Joint Conference on Artificial Intelligence, pages 411-417, 2015.

[Iwata and Shimizu, 2019] Tomoharu Iwata and Hitoshi Shimizu. Neural collective graphical models for estimating spatiotemporal population flow from aggregated data. In AAAI Conference on Artificial Intelligence, 2019.

[Kumar and Zilberstein, 2009] Akshat Kumar and Shlomo Zilberstein. Constraint-based dynamic programming for decentralized pomdps with structured interactions. In International Joint Conference on Autonomous Agents and Multiagent Systems, pages 561-568, 2009.

[Kumar and Zilberstein, 2010] Akshat Kumar and Shlomo Zilberstein. Anytime planning for decentralized POMDPs using expectation maximization. In Conference on Uncertainty in Artificial Intelligence, pages 294-301, 2010.

[Kumar et al., 2011] Akshat Kumar, Shlomo Zilberstein, and Marc Toussaint. Scalable multiagent planning using probabilistic inference. In Proceedings of the Twenty-Second International Joint Conference on Artificial Intelligence, pages 2140-2146, Barcelona, Spain, 2011.

[Kumar et al., 2013] Akshat Kumar, Daniel Sheldon, and Biplav Srivastava. Collective diffusion over networks: Models and inference. In Conference on Uncertainty in Artificial Intelligence, 2013.

[Kumar et al., 2015] Akshat Kumar, Shlomo Zilberstein, and Marc Toussaint. Probabilistic inference techniques for scalable multiagent decision making. Journal of Artificial Intelligence Research, 53:223-270, 2015.

[Kumar et al., 2016] Akshat Kumar, Hala Mostafa, and Shlomo Zilberstein. Dual formulations for optimizing Dec-POMDP controllers. In International Conference on Automated Planning and Scheduling, pages 202-210, 2016.

[Lowe et al., 2017] Ryan Lowe, Yi Wu, Aviv Tamar, Jean Harb, Pieter Abbeel, and Igor Mordatch. Multi-agent actor-critic for mixed cooperative-competitive environments. In Advances in Neural Information Processing Systems, pages 6382-6393, 2017.

[Nair et al., 2005] R. Nair, P. Varakantham, M. Tambe, and M. Yokoo. Networked distributed POMDPs: A synthesis of distributed constraint optimization and POMDPs. In AAAI Conference on Artificial Intelligence, pages 133-139, 2005.

[Nguyen et al., 2016] Duc Thien Nguyen, Akshat Kumar, Hoong Chuin Lau, and Daniel Sheldon. Approximate inference using DC programming for collective graphical models. In International Conference on Artificial Intelligence and Statistics, pages 685-693, 2016.

[Nguyen et al., 2017a] Duc Thien Nguyen, Akshat Kumar, and Hoong Chuin Lau. Collective multiagent sequential decision making under uncertainty. In AAAI Conference on Artificial Intelligence, pages 3036-3043, 2017.

[Nguyen et al., 2017b] Duc Thien Nguyen, Akshat Kumar, and Hoong Chuin Lau. Policy gradient with value function approximation for collective multiagent planning. In Neural Information Processing Systems, pages 4322-4332, 2017.

[Nguyen et al., 2018] Duc Thien Nguyen, Akshat Kumar, and Hoong Chuin Lau. Credit assignment for collective multiagent RL with global rewards. In Advances in Neural Information Processing Systems, pages 8113-8124, 2018.

[Niepert and Van den Broeck, 2014] Mathias Niepert and Guy Van den Broeck. Tractability through exchangeability: A new perspective on efficient probabilistic inference. In AAAI Conference on Artificial Intelligence, pages 2467-2475, July 2014.

[Sheldon and Dietterich, 2011] Daniel R. Sheldon and Thomas G. Dietterich. Collective graphical models. In Neural Information Processing Systems, pages 1161-1169, 2011.

[Sheldon et al., 2013] Daniel Sheldon, Tao Sun, Akshat Kumar, and Thomas G. Dietterich. Approximate inference in collective graphical models. In International Conference on Machine Learning, pages 1004-1012, 2013.

[Singh and Kumar, 2019] Arambam James Singh and Akshat Kumar. Graph based optimization for multiagent cooperation. In International Conference on Autonomous Agents and MultiAgent Systems, pages 1497-1505, 2019.

[Singh et al., 2019] Arambam James Singh, Duc Thien Nguyen, Akshat Kumar, and Hoong Chuin Lau. Multiagent decision making for maritime traffic management. In AAAI Conference on Artificial Intelligence, 2019.

[Spaan and Melo, 2008] Matthijs T. J. Spaan and Francisco S. Melo. Interaction-driven Markov games for decentralized multiagent planning under uncertainty. In International COnference on Autonomous Agents and Multi Agent Systems, pages 525-532, 2008.

[Sun et al., 2015] Tao Sun, Daniel Sheldon, and Akshat Kumar. Message passing for collective graphical models. In International Conference on Machine Learning, pages 853-861, 2015.

[Toussaint and Storkey, 2006] Marc Toussaint and Amos J. Storkey. Probabilistic inference for solving discrete and continuous state markov decision processes. In International Conference on Machine Learning, pages 945-952, 2006.

[Witwicki and Durfee, 2010] Stefan J. Witwicki and Edmund H. Durfee. Influence-based policy abstraction for weakly-coupled Dec-POMDPs. In International Conference on Automated Planning and Scheduling, pages 185-192, 2010. 